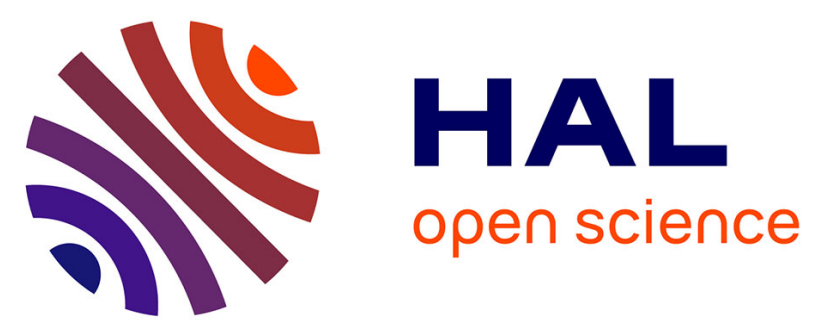

\title{
A New Cycle-Slip Detection Algorithm for Network RTK Using Optimal Dual-Frequency Carrier-Phase Combinations
}

\author{
Donguk Kim, Junesol Song, Sunkyoung Yu, Changdon Kee
}

\section{To cite this version:}

Donguk Kim, Junesol Song, Sunkyoung Yu, Changdon Kee. A New Cycle-Slip Detection Algorithm for Network RTK Using Optimal Dual-Frequency Carrier-Phase Combinations. ITM 2019,20th International Technical Meeting of The Institute of Navigation, Jan 2019, Reston, United States. pp.554-567, 10.33012/2019.16710 . hal-02191101

\section{HAL Id: hal-02191101 \\ https://hal-enac.archives-ouvertes.fr/hal-02191101}

Submitted on 24 Jul 2019

HAL is a multi-disciplinary open access archive for the deposit and dissemination of scientific research documents, whether they are published or not. The documents may come from teaching and research institutions in France or abroad, or from public or private research centers.
L'archive ouverte pluridisciplinaire HAL, est destinée au dépôt et à la diffusion de documents scientifiques de niveau recherche, publiés ou non, émanant des établissements d'enseignement et de recherche français ou étrangers, des laboratoires publics ou privés. 


\title{
A New Cycle-Slip Detection Algorithm for Network RTK Using Optimal Dual-Frequency Carrier-Phase Combinations
}

\author{
Donguk Kim ${ }^{1}$, Junesol Song ${ }^{2}$, Sunkyoung $\mathrm{Yu}^{1}$, Changdon $\mathrm{Kee}^{1^{*}}$ \\ ${ }^{1}$ School of Mechanical and Aerospace Engineering and the SNU-IAMD, \\ Seoul National University, Republic of Korea \\ ${ }^{2}$ Ecole Nationale de l'Aviation Civile (ENAC), France
}

\section{BIOGRAPHY (IES)}

Donguk Kim is a Ph.D. student in the School of Mechanical and Aerospace Engineering at Seoul National University, Republic of Korea. He received the B.S. degree from Seoul National University in 2013. His research interests include Satellite Based Augmentation System (SBAS), network Real-Time Kinematic (RTK), and carrier-phase based algorithms for highprecision navigation system.

Dr. Junesol Song is a postdoctoral fellow in the SIGnal processing and NAVigation (SIGNAV) research group at Ecole Nationale de l'Aviation Civile (ENAC), France. She received B.S. and Ph.D. degrees from Seoul National University, South Korea. Her research interests include carrier phase based algorithm and positioning, and signal quality monitoring in Ground Based Augmentation System (GBAS).

Sunkyoung Yu is a Ph.D. candidate of GNSS Lab. in the School of Mechanical and Aerospace Engineering at Seoul National University. She received B.S. degree in mechanical and aerospace engineering from the same university. Her research interests are precise orbit determination and orbit propagation.

Prof. Changdon Kee is a Professor in the School of Mechanical and Aerospace Engineering at Seoul National University (SNU), South Korea and supervises SNU GNSS Lab (SNUGL, http://gnss.snu.ac.kr). He received B.S. and M.S. degrees from Seoul National University and a Ph.D. degree from Stanford University. He served as a Technical Advisor to the Federal Aviation Administration (FAA) on the Wide Area Augmentation System (WAAS) in 1994. Prof. Kee currently serves as a Technical Advisor for Korea Civil Aviation Safety Authority (KCASA) and Ministry of Public Administration and Security (MOPAS). He also served as a President of Korean Institute of Navigation. He has more than 20 years of GNSS and flight control research experiences. He is corresponding author of this paper. (Correspondence: kee@snu.ac.kr)

\begin{abstract}
Many strategies for treating dual-frequency cycle slip have been studied over the years; however, the conventional method using the Melbourne-Wübbena (MW) combination is vulnerable to pseudorange multipath effects. In this paper, we propose a new detection algorithm of dual-frequency cycle slip using only carrier-phase stationary observations for the network real-time kinematic (RTK) system which generates high-precision corrections for user. Two independent and complementary carrierphase combinations, called the ionospheric negative (IN) and ionospheric positive (IP) combinations in this paper, are employed for avoiding insensitive pairs. They can successfully detect all of the cycle slips since two L1/L2 combinations combine cycle slips with opposite signs for uniquely detecting insensitive pairs. We verified that the actual error distributions under severe ionospheric storm of these monitoring values can be sufficiently bounded by the normal Gaussian distribution from a theoretical analysis. Consequently, we demonstrated that the proposed method ensures high-integrity performance with a probability of missed detection of $7.5 \times 10^{-9}$. under a desired false-alarm probability of $10^{-5}$. In addition, the IN and IP combination shows the best detection performance than the other linear combinations such as ionosphere-free, wide-lane, and narrow-lane. Through an algorithm verification test using actual data collected under a severe ionospheric storm, we confirmed that all artificially inserted cycle slips are successfully detected. In conclusion, the proposed method is confirmed to be effective for handling dual-frequency cycle slips for network RTK system.
\end{abstract}




\section{INTRODUCTION}

A real-time kinematic (RTK) is a carrier-phase-based positioning system for obtaining cm-level accuracy; however, the conventional single-baseline RTK has been constrained to short-baselines under $10 \mathrm{~km}$. Over the past few decades, networkbased RTK techniques have been studied to enlarge RTK coverage per reference station from medium to long-baseline and maintain high-precision accuracy at the same time. Among them, master-auxiliary concept (MAC) based compact network RTK proposed by GNSS Laboratory at Seoul National University is considered as a candidate solution for land vehicle user because it provides $\mathrm{cm}$-level positioning service with fast ambiguity resolution under extremely low-rate data link [1]-[3].

One of the most important issues in the network RTK is how to generate carrier-phase based corrections without cycle slips, which is an instantaneous jump of an integer number of a cycle. A cycle slip occurs unexpectedly when the receiver's phaselocked loop (PLL) has a loss of lock during a temporary signal blockage or an ionospheric scintillation [4]-[6]. The cycle slip must be handled at the data pre-processing since it can seriously affect the quality of high-precision corrections generated after the integer ambiguity resolution.

A number of processing methods for handling cycle slip have been studied over the years. In particular, the cycle-slip detection and repair methods using two linear combinations of dual-frequency observations were widely used for static and even dynamic users [4], [7]-[10]. These popular methods employ two complementary geometry-free linear combinations, the Melbourne-Wübbena (MW) combination and ionosphere combination, since the combined dual-frequency cycle slip can be close to zero and cannot be detected using only one combination. For example, the same size of cycle slips on L1 and L2 frequencies such as $(10,10)$ cannot be noticed on the MW combination, but it has a detectable value on the ionosphere combination. Similarly, the special cycle-slip pair $(77,60)$, which does not influence the ionosphere combination, is detected by the MW combination. We call these special cycle-slip pairs insensitive pairs [10], [11].

Most dual-frequency insensitive cycle-slip pairs can be detected by the strategy using the MW combination and ionosphere combination. However, this conventional method has critical limitations when detecting small size of cycle-slip pairs. First of all, the MW combination is vulnerable to pseudorange multipath effects even if a smoothing or averaging techniques such as a Hatch filter and low-pass filter is applied. In other words, these techniques cannot be processed instantaneously and might fail to detect small cycle slips under high-multipath conditions [10], [11]. Second, the ionosphere combination is affected by severe ionospheric activity such as geomagnetic storm because the remaining trend bias of the ionospheric variation makes it difficult to detect small cycle slips [10], [12], [13]. For example, the cycle-slip pair $(5,4)$ failed to detect by the MW combination and even ionosphere combination [3]. The probability of occurrence of these insensitive pairs is incredibly small, but it should be make a critical integrity threat when we solve the integer ambiguity of carrier-phase observations. To overcome these limitations, cycle-slip detection algorithms using triple-frequency signals or additional sensor aiding such as inertial navigation system (INS) are being emphasized and studied [12]-[15]. However, most of current receivers and GNSS satellites only provide dual-frequency signals. That is, the demand for a cycle-slip detection method based on only dual-frequency signals remains very strong.

Considering the limitations discussed above, we proposed a new algorithm for cycle-slip detection for reference stations of the network RTK system using the only dual-frequency carrier-phase observations without pseudorange. We introduced two independent and complementary ionosphere combinations of carrier-phase observations: one is the geometry-free ionosphere combination usually used in cycle-slip detection, and the other is a new geometry-based ionosphere combination to replace the MW combination based on pseudorange. We named these combinations the ionospheric negative (IN) and ionospheric positive (IP) combination, respectively [16]. Song and Kee demonstrated that there is no additional geometry-free carrier-phase combination to replace the MW combination for dynamic users [11]; however, geometry-based combinations can be employed in static permanent stations of the network RTK for generating reliable high-precision corrections. Because the IP combination constructs the combined cycle slips with positive signs, it can detect a small cycle-slip pairs more efficiently, which cannot be detected by the IN combination. We already demonstrated the IP combination can be replaced the conventional MW combination, and the proposed method ensure very small missed detection probability [16].

In this paper, we briefly introduce the new cycle-slip detection algorithm with the IN and IP combinations, and we verify that the IN and IP combinations are optimal dual-frequency carrier-phase combinations for cycle-slip detection. First of all, we theoretically analyze the noise level of various dual-frequency carrier-phase combinations and verify that the actual error distributions are properly bounded to the normal Gaussian distribution even in severe ionospheric storms. This analysis of statistical error propagation and bounding is very important because it is impossible to collect an extremely large number of data sets for reliable integrity statistics [17]-[19]. Next, we evaluate the probability of missed detection, which is the most significant index for evaluating performance of a detection algorithm, under the desired false alarm probability. Many former studies overlooked the probability of missed detection and only analyzed whether the size of the cycle slip was greater than the 
threshold; however, it is necessary to demonstrate the performance of the proposed method quantitatively for conserving integrity. Then, we analyze and choose two optimal linear-combinations that minimize a probability of missed detection. Finally, we conduct an algorithm verification test with actual observations under a severe ionospheric storm conditions. We inserted the simulated cycle slips artificially into the raw data and checked whether all of them are successfully detected.

\section{A NEW CYCLE-SLIP DETECTION ALGORITHM FOR NETWORK RTK}

\section{Cycle-Slip Detection with Ionospheric Negative and Positive Combinations}

Figure 1 shows a block diagram of the overall cycle-slip detection and compensation algorithm for the network RTK system that we proposed previously [16]. In this section, we briefly introduce the cycle-slip detection part of the proposed algorithm.

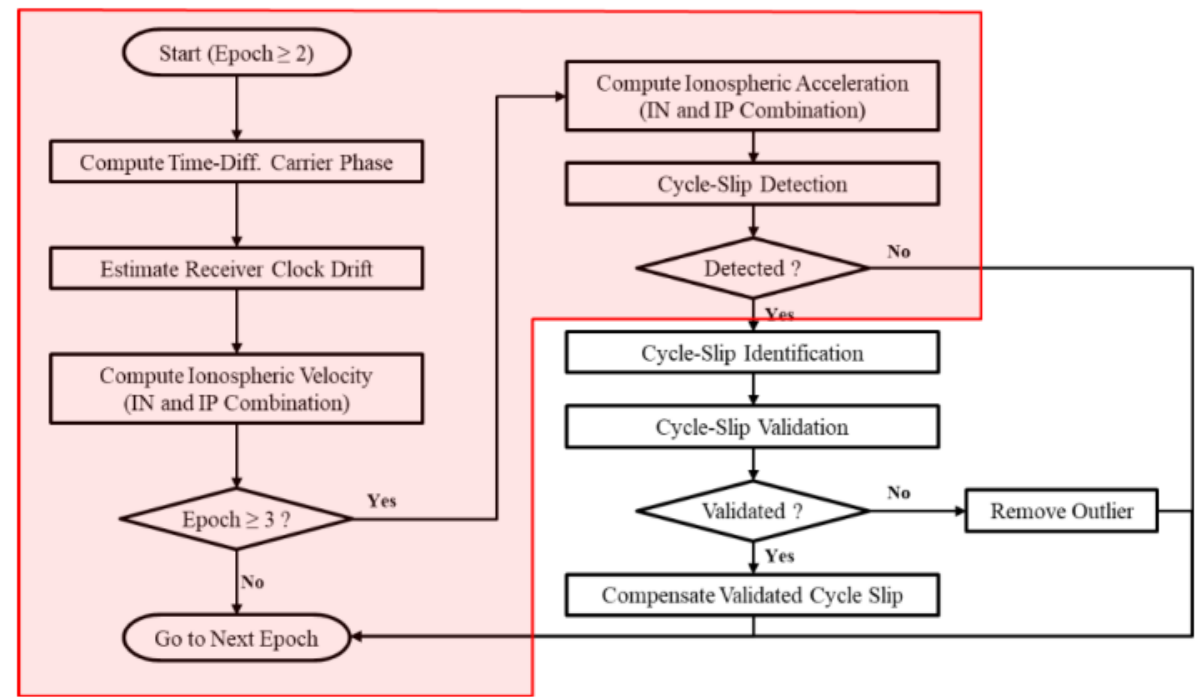

Figure 1. Block diagram of the overall cycle-slip detection and compensation algorithm

The network RTK system generates high-precision corrections based on single-differenced carrier-phase observations between reference stations. Therefore, L1 and L2 carrier-phase observations should be handled as time-differences (TDs) and singledifferences (SDs) between static receivers in order to detect cycle slips. By single-differencing the observations between receivers, satellite clock biases are eliminated. The TDSD L1 and L2 carrier-phase observations of reference stations are defined as follows:

$$
\begin{aligned}
& \delta_{T} \Delta \phi_{1}=\lambda_{1} \cdot C S_{1}-\delta_{T} \Delta I+\delta_{T} \Delta L+\delta_{T} \Delta B+\varepsilon_{\delta_{T} \Delta \phi_{1}} \\
& \delta_{T} \Delta \phi_{2}=\lambda_{2} \cdot C S_{2}-\gamma \cdot \delta_{T} \Delta I+\delta_{T} \Delta L+\delta_{T} \Delta B+\varepsilon_{\delta_{T} \Delta \phi_{2}}
\end{aligned}
$$

where the symbols $\delta_{T}$ and $\Delta$ indicate the TD and SD operators, respectively; $\phi$ is the measured carrier phase; the subscripts " 1 " and " 2 " represent the L1 and L2 frequencies, respectively; $\lambda$ is the wavelength; CS is an integer cycle slip that may exist in phase; $I$ is the ionospheric slant delay on the L1 frequency; and $\gamma$ is the square of the ratio of the L1 and L2 frequencies. In this process, we can eliminate the geometric distance using satellite ephemeris since we know the precise position of reference stations. Furthermore, the tropospheric slant delay can be reduced by nominal model. The symbol $L$ denotes the sum of the geometric distance error and the modeling error of tropospheric slant delay. $B$ is the receiver clock offset, and $\varepsilon$ is the receiver noise of observation.

Most of the conventional cycle-slip detection methods usually used geometry-free linear-combination as monitoring values for applying dynamic users. That is, there was no need to consider the time variation of the receiver clock, which would interfere with the detection of cycle slips. On the other hand, the geometry-based linear combinations should be eliminate the receiver clock drift to consider as monitoring values of cycle-slip detection. The receiver clock drift of the reference station, which has highly stable oscillator, can be estimated precisely from the average value of each satellite's ionosphere-free carrier-phase combination. The details of the receiver clock estimation method have been described by D. Kim, et al. [16]. The TDSD L1 and L2 carrier-phase observations after the receiver clock drift compensation can be expressed as follows: 


$$
\begin{aligned}
& \delta_{T} \Delta \tilde{\phi}_{1}=\delta_{T} \Delta \phi_{1}-\delta_{T} \Delta \hat{B}=\lambda_{1} \cdot C S_{1}-\delta_{T} \Delta I+\delta_{T} \Delta L+\varepsilon_{\delta_{T} \Delta \tilde{\phi}_{1}} \\
& \delta_{T} \Delta \tilde{\phi}_{2}=\delta_{T} \Delta \phi_{2}-\delta_{T} \Delta \hat{B}=\lambda_{2} \cdot C S_{2}-\gamma \cdot \delta_{T} \Delta I+\delta_{T} \Delta L+\varepsilon_{\delta_{T} \Delta \tilde{\phi}_{2}}
\end{aligned}
$$

where the symbols $\delta_{T} \Delta \hat{B}$ indicate the estimated SD receiver clock drift. We call Equation (2) the TDSD carrier-phase residual. In this sub-section, we describe two L1/L2 linear combinations of TDSD carrier-phase residual for obtaining the monitoring values (MVs) of cycle-slip detection. The first observation is the geometry-free ionosphere combination, which is widely used to detect dual-frequency cycle slips [4], [7]-[10]. In this paper, we named it the ionosphere negative (IN) combination because L1 and L2 cycle slips are combined with negative signs.

$$
\begin{aligned}
& \delta_{T} \Delta I^{-}=b_{1}^{-} \cdot \delta_{T} \Delta \tilde{\phi}_{1}+b_{2}^{-} \cdot \delta_{T} \Delta \tilde{\phi}_{2}=\delta_{T} \Delta I+\frac{1}{\gamma-1}\left(\lambda_{1} \cdot C S_{1}-\lambda_{2} \cdot C S_{2}\right)+\varepsilon_{\delta_{T} \Delta I^{-}} \\
& \text {where } b_{1}^{-}=\frac{1}{\gamma-1}, b_{2}^{-}=\frac{-1}{\gamma-1}
\end{aligned}
$$

In general, the size of the combined cycle slips in the IN combination is much greater than the temporal change of the ionospheric slant delay and the measured noise; however, the combined cycle slip can be almost zero even if the sizes of L1 and L2 cycle slips are not small. For example, the special cycle-slip pair $(77,60)$ makes combined cycle slip exactly zero. Therefore, we need a complementary linear combination that can detect insensitive cycle-slip pairs in the IN combination. We proposed the another ionosphere combination, named ionosphere positive (IP) combination, that couples the L1 and L2 cycle slips with positive signs [16], [20].

$$
\begin{aligned}
& \delta_{T} \Delta I^{+}=b_{1}^{+} \cdot \delta_{T} \Delta \tilde{\phi}_{1}+b_{2}^{+} \cdot \delta_{T} \Delta \tilde{\phi}_{2}=-\delta_{T} \Delta I+\frac{1}{2}\left(1+\frac{1}{\gamma}\right) \delta_{T} \Delta L+\frac{1}{2}\left(\lambda_{1} \cdot C S_{1}+\frac{1}{\gamma} \lambda_{2} \cdot C S_{2}\right)+\varepsilon_{\delta_{T} \Delta I^{+}} \\
& \text {where } b_{1}^{+}=\frac{1}{2}, b_{2}^{+}=\frac{1}{2 \gamma}
\end{aligned}
$$

The IP combination is not geometry-free combination; however, the temporal variations of satellite orbit error and tropospheric slant delay are less than 1 and $0.2 \mathrm{~mm} / \mathrm{s}$, respectively [2], [21]. It is sufficiently negligible value than typically measured carrier-phase noise. Since the IN and IP combinations combine cycle slips with different signs and are complementary to each other, all cycle slips can be successfully detected under the sufficiently slow change of ionospheric delay. However, the variation of ionospheric delay cannot be ignored for detecting small size cycle slips, because it can be greater than $2 \mathrm{~cm} / \mathrm{s}$ under high ionospheric storm conditions [2], [21]. Thus, we employ ionospheric acceleration, which is the second-order time derivative of ionospheric slant delay, as MVs. Many former research considered is as the MV for robust cycle-slip detection under high-ionospheric activity since the trend of the ionospheric acceleration is sufficiently small [10], [12]. The two linear combinations of the ionospheric acceleration can be expressed as follows:

$$
\begin{gathered}
\delta_{T}^{2} \Delta I^{-}=\delta_{T}^{2} \Delta I+\frac{1}{\gamma-1}\left(\lambda_{1} \cdot C S_{1}-\lambda_{2} \cdot C S_{2}\right)+\varepsilon_{\delta_{T}^{2} \Delta I^{-}} \\
\delta_{T}^{2} \Delta I^{+}=-\delta_{T}^{2} \Delta I+\frac{1}{2}\left(1+\frac{1}{\gamma}\right) \delta_{T}^{2} \Delta L+\frac{1}{2}\left(\lambda_{1} \cdot C S_{1}+\frac{1}{\gamma} \lambda_{2} \cdot C S_{2}\right)+\varepsilon_{\delta_{T}^{2} \Delta I^{+}}
\end{gathered}
$$

Equation (5) corresponds to the IN combination, and Equation (6) corresponds to the IP combination. The symbol $\delta_{T}^{2}$ represents the second-order TD operator. These MVs are considered to have only receiver noise with negligible biases under the non-cycle slip hypothesis. Therefore, the cycle slips can be detected when the following condition is satisfied:

$$
\begin{aligned}
& \left|M V^{-}\right| \triangleq\left|\delta_{T}^{2} \Delta I^{-}\right|>T^{-} \\
\text {or } \quad & \left|M V^{+}\right| \triangleq\left|\delta_{T}^{2} \Delta I^{+}\right|>T^{+}
\end{aligned}
$$

where $T^{-}$and $T^{*}$ indicate the thresholds for each MV. We will discuss how to determine thresholds for the detection criterion in more detail in next section. 


\section{General Form of Linear Combinations for Cycle-Slip Detection and Their Theoretical Noise Analysis}

In order to reduce trends in ionospheric velocity, we consider second-order time-derivative of carries-phase combinations as well as the IN and IP combination described above. The general form of them can be expressed by Equation (8). The symbols $\beta_{1}$ and $\beta_{2}$ indicate linear combination coefficient of each frequency. We called this general form as ionospheric acceleration combination.

$$
\begin{aligned}
& \begin{aligned}
\delta_{T}^{2} \Delta I_{\left(\beta_{1}, \beta_{2}\right)} & =\beta_{1} \cdot \delta_{T}^{2} \Delta \tilde{\phi}_{1}+\beta_{2} \cdot \delta_{T}^{2} \Delta \tilde{\phi}_{2} \\
& =\left(\beta_{1} \lambda_{1} \cdot C S_{1}+\beta_{2} \lambda_{2} \cdot C S_{2}\right)-\left(\beta_{1}+\beta_{2} \gamma\right) \delta_{T}^{2} \Delta I+\left(\beta_{1}+\beta_{2}\right) \delta_{T}^{2} \Delta L+\left(\beta_{1} \varepsilon_{\delta_{T}^{2} \Delta \tilde{\phi}_{1}}+\beta_{2} \varepsilon_{\delta_{T}^{2} \Delta \tilde{\phi}_{2}}\right)
\end{aligned} \\
& \text { where } \quad \beta_{1}+\beta_{2} \gamma \in\{0,+1,-1\}
\end{aligned}
$$

All ionospheric acceleration combinations would be candidates for MVs if they can be assumed to be a normal Gaussian distributions under the non-cycle slip hypothesis. Unfortunately, the actual measured data does not have a perfect Gaussian distribution, because of, e.g., the influence of carrier-phase multipath effects, clock estimation errors, and the remaining bias of ionospheric delay. Therefore, the standard deviation of the ionospheric acceleration combinations must be carefully and conservatively determined for sufficiently bounding the tails of the actual error distribution [17]-[19]. In other words, we should theoretically analyze the statistics of them considering the worst possible scenario. The theoretical variance of the ionospheric acceleration combinations can be calculated as following equation. We consider the variance of the estimated receiver clock is uncorrelated with carrier-phase measurement noise, and we assume the L1 and L2 carrier phase have the same noise level, which is typically measured as approximately $\sigma_{\phi_{1}} \approx \sigma_{\phi_{2}} \approx 2 \mathrm{~mm}$.

$$
\begin{aligned}
\sigma^{2}\left(\delta_{T}^{2} \Delta I_{\left(\beta_{1}, \beta_{2}\right)}\right) & =\sigma^{2}\left(\beta_{1} \varepsilon_{\delta_{T}^{2} \Delta \bar{\phi}_{1}}+\beta_{2} \varepsilon_{\delta_{T}^{2} \Delta \bar{\phi}_{2}}\right) \\
& =\sigma^{2}\left\{\beta_{1}\left(\varepsilon_{\delta_{T}^{2} \Delta \phi_{1}}-\varepsilon_{\delta_{T}^{2} \Delta \hat{B}}\right)+\beta_{2}\left(\varepsilon_{\delta_{T}^{2} \Delta \phi_{2}}-\varepsilon_{\delta_{T}^{2} \Delta \hat{B}}\right)\right\} \\
& =\left(\beta_{1}^{2}+\beta_{2}^{2}\right) \cdot \sigma_{\delta_{T}^{2} \Delta \phi}^{2}+\left(\beta_{1}+\beta_{2}\right)^{2} \cdot \sigma^{2}\left(\delta_{T}^{2} \Delta \hat{B}\right)
\end{aligned}
$$

The estimation variance of the receiver clock, which is the average value of the ionosphere-free (IF) combination, can be expressed as follows:

$$
\begin{gathered}
\sigma^{2}\left(\delta_{T}^{2} \Delta \hat{B}\right)=\sigma^{2}\left(\frac{1}{m} \sum^{m} \delta_{T}^{2} \Delta \phi_{I F}\right)=\frac{a_{1}^{2}+a_{2}^{2}}{m} \cdot \sigma_{\delta_{T}^{2} \Delta \phi}^{2} \\
\text { where } \quad \delta_{T}^{2} \Delta \phi_{I F}=a_{1} \cdot \delta_{T}^{2} \Delta \phi_{1}+a_{2} \cdot \delta_{T}^{2} \Delta \phi_{2} \\
a_{1}=\frac{\gamma}{\gamma-1}, a_{2}=\frac{-1}{\gamma-1}
\end{gathered}
$$

The symbol $m$ is the number of observations used for clock estimation, and the variance is mathematically largest when $m=1$. Therefore, the variance of the ionospheric acceleration combination becomes maximum as follows:

$$
\begin{aligned}
& \sigma_{\max }^{2}\left(\delta_{T}^{2} \Delta I_{\left(\beta_{1}, \beta_{2}\right)}\right)=\left\{\left(\beta_{1}^{2}+\beta_{2}^{2}\right)+\left(\beta_{1}+\beta_{2}\right)^{2} \cdot \frac{a_{1}^{2}+a_{2}^{2}}{m}\right\} \cdot \sigma_{\delta_{T}^{2} \Delta \phi}^{2} \triangleq \operatorname{func}\left(\beta_{1}, \beta_{2}\right) \cdot \sigma_{\delta_{T}^{2} \Delta \phi}^{2} \\
& \text { where } m=1
\end{aligned}
$$

Since the measurement noise of the carrier-phase observations can be regarded as uncorrelated in time and each observation, the variance of second-order TD observations is 6 times (i.e., $\sigma_{\sigma_{f}^{2} \phi}^{2}=6 \cdot \sigma_{\phi}^{2}$ ), and SD observations is twice (i.e., $\sigma_{\Delta \phi}^{2}=2 \cdot \sigma_{\phi}^{2}$ ) that of the un-difference observations. Finally, the maximum standard deviation of the ionospheric acceleration combination is theoretically determined as follows:

$$
\sigma_{\left(\beta_{1}, \beta_{2}\right)} \triangleq \sigma_{\max }\left(\delta_{T}^{2} \Delta I_{\left(\beta_{1}, \beta_{2}\right)}\right)=\sqrt{\operatorname{func}\left(\beta_{1}, \beta_{2}\right)} \cdot \sqrt{6} \cdot \sqrt{2} \cdot \sigma_{\phi}
$$

Table 1 summarizes the expression and noise level of typical dual-frequency combinations which are candidates for MVs. 
Table 1. Typical ionospheric acceleration combination of dual-frequency carrier phase

\begin{tabular}{|c|c|c|c|}
\hline Combination Name & Coefficients $\left(\beta_{1}, \beta_{2}\right)$ & Expression & Noise Level (Max) \\
\hline $\begin{array}{l}\text { Ionospheric Negative } \\
\text { (IN) }\end{array}$ & $\left(\frac{1}{\gamma-1}, \frac{-1}{\gamma-1}\right)$ & $\delta_{T}^{2} \Delta I^{-}=\delta_{T}^{2} \Delta I+\frac{1}{\gamma-1}\left(\lambda_{1} \cdot C S_{1}-\lambda_{2} \cdot C S_{2}\right)+\varepsilon_{\delta_{\tau}^{*} \Delta I^{-}}$ & $\begin{aligned} \sigma_{M V^{\prime}} & =7.57 \cdot \sigma_{\phi} \\
& =15.1 \mathrm{~mm} / \mathrm{s}^{2}\end{aligned}$ \\
\hline $\begin{array}{l}\text { Ionospheric Positive } \\
\text { (IP) }\end{array}$ & $\left(\frac{1}{2}, \frac{1}{2 \gamma}\right)$ & $\delta_{T}^{2} \Delta I^{+}=-\delta_{T}^{2} \Delta I+\frac{1}{2}\left(1+\frac{1}{\gamma}\right) \delta_{T}^{2} \Delta L+\frac{1}{2}\left(\lambda_{1} \cdot C S_{1}+\frac{1}{\gamma} \lambda_{2} \cdot C S_{2}\right)+\varepsilon_{\delta \delta_{T}^{2} \Delta^{+}}$ & $\begin{aligned} \sigma_{M N^{+}} & =8.53 \cdot \sigma_{\phi} \\
& =17.1 \mathrm{~mm} / \mathrm{s}^{2}\end{aligned}$ \\
\hline $\begin{array}{l}\text { Ionospheric-Free } \\
\text { (IF) }\end{array}$ & $\left(\frac{\gamma}{\gamma-1}, \frac{-1}{\gamma-1}\right)$ & $\delta_{T}^{2} \Delta I_{I F}=\delta_{T}^{2} \Delta L+\frac{1}{\gamma-1}\left(\gamma \lambda_{1} \cdot C S_{1}-\lambda_{2} \cdot C S_{2}\right)+\varepsilon_{\delta_{T}^{2} \Delta I_{I F}}$ & $\begin{aligned} \sigma_{I F} & =14.59 \cdot \sigma_{\phi} \\
& =29.2 \mathrm{~mm} / \mathrm{s}^{2}\end{aligned}$ \\
\hline $\begin{array}{l}\text { Wide-Lane } \\
\text { (WL) }\end{array}$ & $\left(\frac{f_{1}}{f_{1}-f_{2}} \cdot \frac{f_{2}}{f_{1}}, \frac{-f_{2}}{f_{1}-f_{2}} \cdot \frac{f_{2}}{f_{1}}\right)$ & $\delta_{T}^{2} \Delta I_{m Z}=\delta_{T}^{2} \Delta I+\left(\frac{f_{2}}{f_{1}}\right) \delta_{T}^{2} \Delta L+\frac{c}{f_{1}-f_{2}} \cdot \frac{f_{2}}{f_{1}}\left(C S_{1}-C S_{2}\right)+\varepsilon_{b_{z}^{2} \Delta \Delta_{m Z}}$ & $\begin{aligned} \sigma_{m L} & =17.46 \cdot \sigma_{\phi} \\
& =34.9 \mathrm{~mm} / \mathrm{s}^{2}\end{aligned}$ \\
\hline $\begin{array}{l}\text { Narrow-Lane } \\
\quad(\mathrm{NL})\end{array}$ & $\left(\frac{f_{1}}{f_{1}+f_{2}} \cdot \frac{f_{2}}{f_{1}}, \frac{f_{2}}{f_{1}+f_{2}} \cdot \frac{f_{2}}{f_{1}}\right)$ & $\delta_{T}^{2} \Delta I_{N I}=-\delta_{T}^{2} \Delta I+\left(\frac{f_{2}}{f_{1}}\right) \delta_{T}^{2} \Delta L+\frac{c}{f_{1}+f_{2}} \cdot \frac{f_{2}}{f_{1}}\left(C S_{1}+C S_{2}\right)+\varepsilon_{\delta_{F}^{2} \Delta \Delta_{N I}}$ & $\begin{aligned} \sigma_{N L} & =8.27 \cdot \sigma_{\phi} \\
& =16.5 \mathrm{~mm} / \mathrm{s}^{2}\end{aligned}$ \\
\hline
\end{tabular}

\section{Actual Error Distribution of Monitoring Values under Sever Ionospheric Storm Conditions}

In this sub-section, we analyze the actual error distribution of candidates of MVs by using the observed data under various ionospheric storm conditions. A severe geomagnetic storm occurred on 17 March 2015. During this storm, the geomagnetic Kp index reached 8-, and the Dst index dropped to $-223 \mathrm{nT}$. Figure 2(a) shows the time history of the geomagnetic indices Kp and Dst for 16-18 March 2015. The ionosphere was disturbed violently from quiet to severe for these three days in the mid-latitude including the Korean region. We collected GPS carrier-phase measurements with 1-s intervals for the three days from the six reference stations of the National Geographic Information Institute (NGII) in Korea. All the stations have a Trimble NetR9 receiver connected with a TRM59800 antenna, and their locations are shown in Figure 2(b). We pre-processed cycle slips and outliers in the collected data by using Bernese GNSS software to obtain nominal monitoring values for each ionospheric acceleration combination under non-cycle-slip hypothesis.
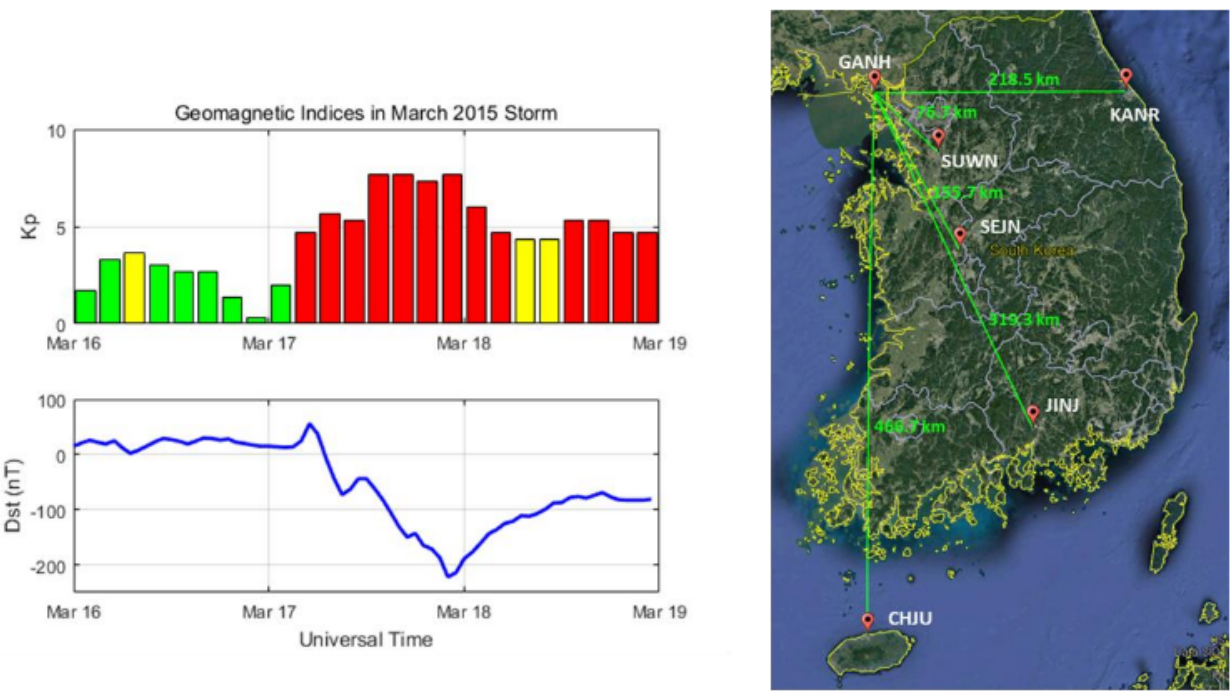

(a)

(b)

Figure 2. (a) The geomagnetic indices (Kp and Dst) during 16-18 March 2015;

(b) Locations of the six reference stations of NGII in Korea

We especially validate that the "fat tails" (i.e., non-Gaussian edge) of the actual error distribution are properly bounded to the theoretical error distribution of the MVs that we calculated previous sub-section. The cumulative distribution function (CDF) bounding plot is one of the useful analysis method to examine the tails of actual sample distribution for validating the reliable assumption of the normal Gaussian distribution [17]-[19]. Figure 3 represents the folded CDF bounding plots of the ionospheric acceleration combinations normalized by the standard deviation of experimental data. The plot of a standard CDF has an S-like shape, while the folded CDF plots, which folds the top-half of the standard CDF graph over, has mountain shape [16]. In order to show the tail shape of the distribution more clearly, scale of its vertical axis is logarithmic. The blue curve line represents the actual sample-data CDF, the red line represents the 1-sigma standard CDF, and the yellow line means the 
theoretically determined CDF. We can clearly see that the blue curve line has non-Gaussian tail, which is fatter than the red line, but thinner than the yellow line. This means that the theoretically determined distribution well bounds the actual sample distributions. For example, as shown in Figure 3(a), the probability that the normalized error of actual IN combination (the blue line) exceeds a 15 -sigma value is less than or equal to $10^{-7}$, under the non-cycle-slip hypothesis; however, the theoretical standard deviation is conservatively calculated that the probability of the actual error exceeding a 15 -sigma is less than or equal to about $3.0 \times 10^{-6}$. In conclusion, we demonstrate that the theoretically determined standard deviation of five candidates of MVs are well-bounded at the $10^{-7}$-level probability of the normal Gaussian distribution even under severe storms. Table 2 summarizes the statistics of the distribution of the five linear combinations. The theoretical maximum standard deviation is approximately more three times inflated than the standard deviation of actual data.

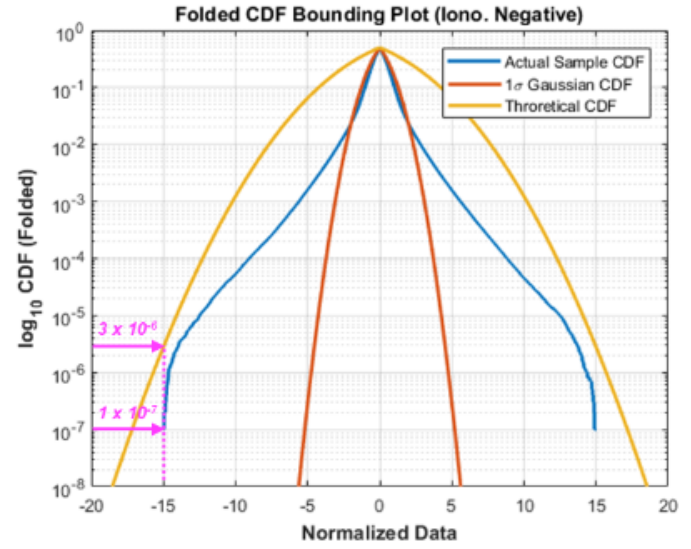

(a)

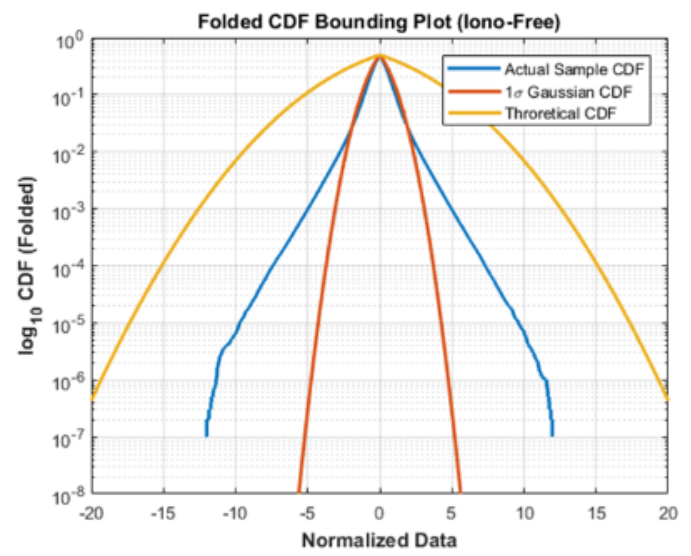

(c)

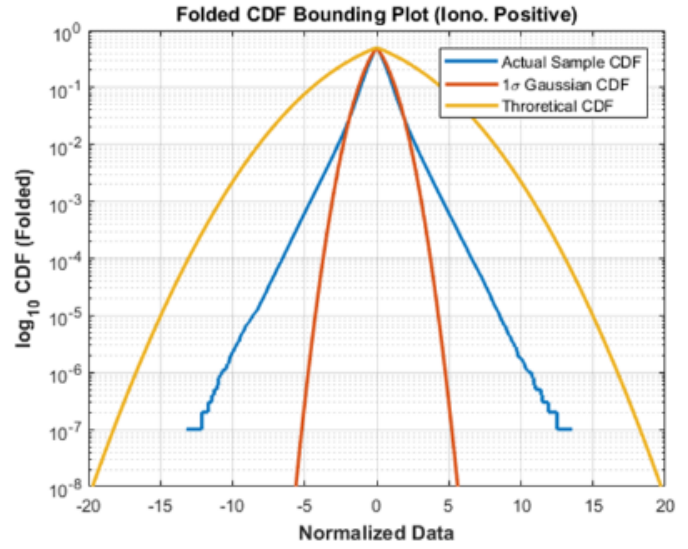

(b)

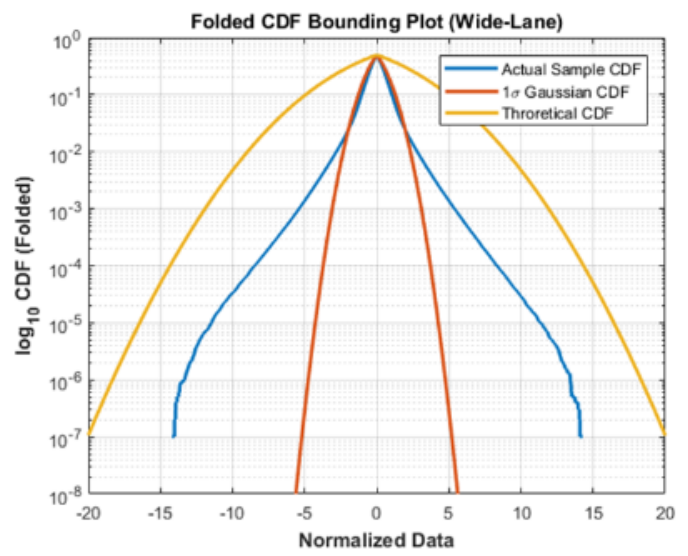

(d)

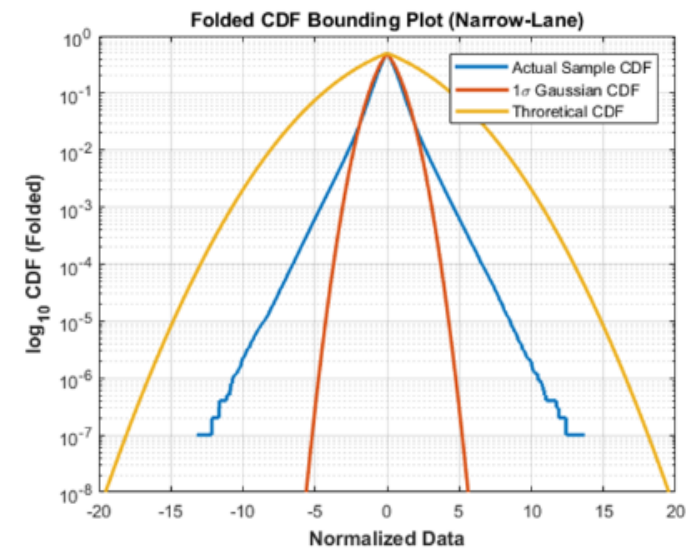

(e)

Figure 3. Folded CDF of nominal monitoring values (note that the scale of its vertical axis is logarithmic):

(a) IN combination; (b) IP combination; (c) IF combination; (d) WL combination; (e) NL combination 
Table 2. Statistics of actual error distribution of the monitoring values

\begin{tabular}{ccccc}
\hline Combination & $\begin{array}{c}\text { Number of } \\
\text { Sample }\end{array}$ & $\begin{array}{c}\text { Actual Sample } \\
\text { Sigma }\end{array}$ & $\begin{array}{c}\text { Theoretical } \\
\text { Sigma }\end{array}$ & $\begin{array}{c}\text { Bounding } \\
\text { Scale Factor }\end{array}$ \\
\hline IN combination & 991,7379 & $4.6 \mathrm{~mm} / \mathrm{s}^{2}$ & $15.1 \mathrm{~mm} / \mathrm{s}^{2}$ & 3.3 \\
IP combination & 991,7379 & $4.9 \mathrm{~mm} / \mathrm{s}^{2}$ & $17.1 \mathrm{~mm} / \mathrm{s}^{2}$ & 3.5 \\
IF combination & 991,7379 & $7.2 \mathrm{~mm} / \mathrm{s}^{2}$ & $29.2 \mathrm{~mm} / \mathrm{s}^{2}$ & 4.1 \\
WL combination & 991,7379 & $9.1 \mathrm{~mm} / \mathrm{s}^{2}$ & $34.9 \mathrm{~mm} / \mathrm{s}^{2}$ & 3.9 \\
NL combination & 991,7379 & $4.8 \mathrm{~mm} / \mathrm{s}^{2}$ & $16.5 \mathrm{~mm} / \mathrm{s}^{2}$ & 3.5 \\
\hline
\end{tabular}

\section{THRESHOLD DETERMINATION SCHEME AND PERFORMANCE EVALUATION}

\section{Probability of False Alarm and Probability of Missed Detection}

In this sub-section, we describe the probability of false alarm and of missed detection, which are the important performance indices of the cycle-slip detection. We already demonstrated that the five independent MVs could be assumed to have an overbounded Gaussian distribution with a zero mean and theoretically determined variances in the cycle-slip-free case (i.e., noncycle-slip hypotheses $H_{0}$ ). When a cycle slip occurs, the PDFs of the MVs are shifted by an amount equal to the size of the combined cycle slip. (i.e., cycle-slip hypotheses $H_{k}$ corresponding to the cycle-slip event $k$ ).

Figure 4 shows the PDF of two independent MVs under the above hypotheses. Considering a given threshold, there is a possibility that the MV exceeds the threshold when a cycle slip does not occur. Such an event is termed as a false alarm (FA). On the other hand, there is the possibility that the MV does not exceed the threshold when cycle slips are actually present. That is, the shifted bias under the cycle-slip hypothesis can be smaller than the given threshold. Such an event is termed as missed detection (MD) [16], [22]. FA and MD can be derived as follows:

Probability of false alarm for each MV:

$$
\begin{aligned}
& P_{F A}^{-}=P\left(\left|M V^{-}\right| \geq T^{-} \mid H_{0}\right)=2\left(1-\Phi\left(\frac{T^{-}}{\sigma_{M V^{-}}}\right)\right) \\
& P_{F A}^{+}=P\left(\left|M V^{+}\right| \geq T^{+} \mid H_{0}\right)=2\left(1-\Phi\left(\frac{T^{+}}{\sigma_{M V^{+}}}\right)\right)
\end{aligned}
$$

Probability of missed detection for each MV:

$$
\begin{gathered}
P_{M D, H_{k}}^{-}=P\left(\left|M V^{-}\right|<T^{-} \mid H_{k}\right)=\Phi\left(\frac{T^{-}-\mu_{k}^{-}}{\sigma_{M V^{-}}}\right) \\
P_{M D, H_{k}}^{+}=P\left(\left|M V^{+}\right|<T^{+} \mid H_{k}\right)=\Phi\left(\frac{T^{+}-\mu_{k}^{+}}{\sigma_{M V^{+}}}\right) \\
\text {where } \Phi(x)=\int_{-\infty}^{x} \frac{1}{\sqrt{2 \pi}} \exp \left(-\frac{1}{2} z^{2}\right) d z
\end{gathered}
$$

Since cycle slips are detected using two complementary and independent MVs, the total probability of FA is determined as the sum of the FA rate of each MV. On the other hand, the total probability of MD can be calculated by the product of the MD rate of each MV.

Total probability of false alarm:

$$
P_{F A}=P\left(\left|M V^{-}\right| \geq T^{-} \text {or }\left|M V^{+}\right| \geq T^{+} \mid H_{0}\right)=P_{F A}^{-}+P_{F A}^{+}
$$

Total probability of missed detection (under the $H_{k}$ hypothesis):

$$
P_{M D, H_{k}}=P\left(\left|M V^{-}\right|<T^{-} \text {and }\left|M V^{+}\right|<T^{+} \mid H_{k}\right)=P_{M D, H_{k}}^{-} \times P_{M D, H_{k}}^{+}
$$




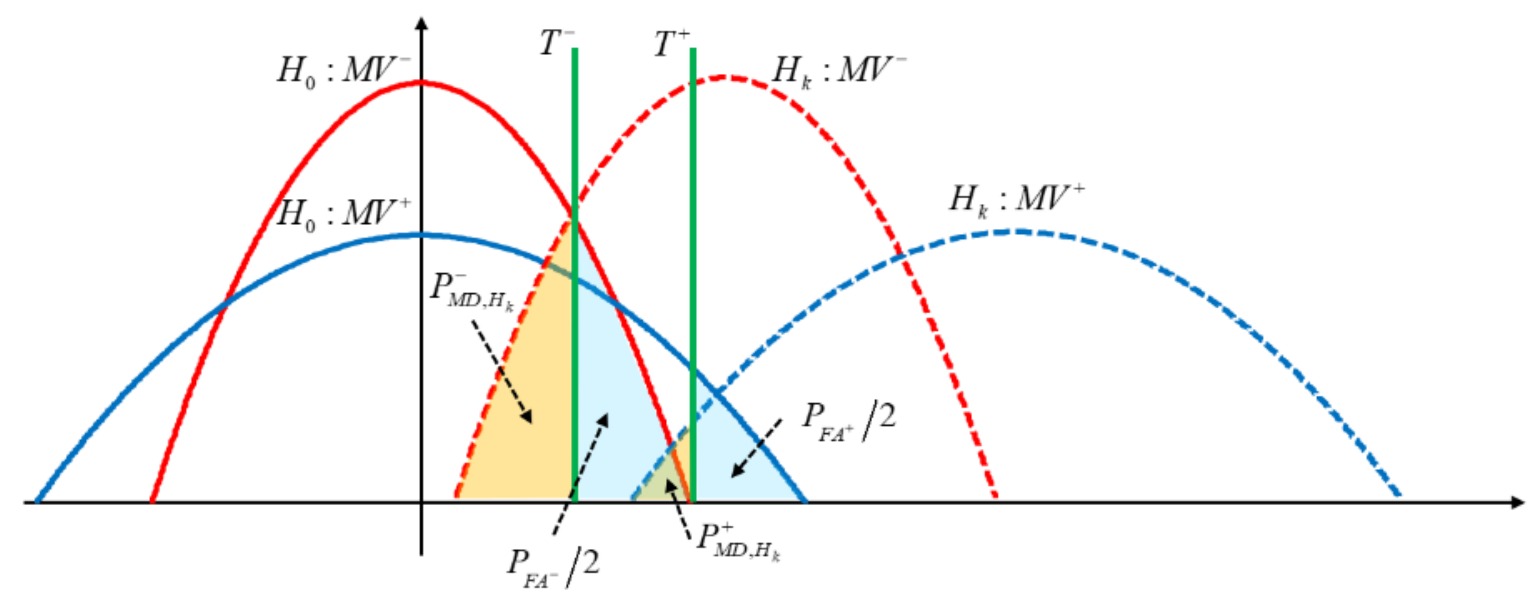

Figure 4. Probability density function of two monitoring values; the region shaded in orange represents the probability of missed detection, and the sky-blue region represents half of the false-alarm probability.

\section{Threshold Determination Scheme}

A good detection algorithm has small probabilities of both FA and MD; however, these probabilities are correlated with each other by the detection threshold. If we determine the threshold to minimize FA rate, the probability of MD should be increased and vice versa. Obviously, a threshold of high-integrity and safe detection should be determined to minimize the probability of $\mathrm{MD}$, which is more critical and important than the FA rate. Therefore, a threshold determine on the basis of a desired FA probability, after that the MD probability is computed by a given threshold. According to the threshold-determination scheme, we allocated the same probability of FA for each MV as $5.0 \times 10^{-6}$. That is, the desired total FA probability is $10^{-5}$. The corresponding confidence level $K_{F A}$ is 4.565 . In conclusion, the proposed cycle-slip detection method be re-expressed with the determined threshold values as follows:

$$
\begin{array}{ll} 
& \left|M V^{-}\right|>T^{-}=K_{F A} \cdot \sigma_{M V^{-}} \\
\text {or } & \left|M V^{+}\right|>T^{+}=K_{F A} \cdot \sigma_{M V^{+}}
\end{array}
$$

\section{Probability of Missed Detection for Insensitive Cycle-Slip Pairs}

Figure 5 shows the L1/L2 insensitive cycle-slip pairs of each combination. We assumed that there is only a bias effect for each cycle-slip pair in detection test domain. The size of the bias must at least be greater than the threshold in order to be detectable. The L1/L2 insensitive cycle-slip pairs of each combination are satisfied as follows:

Insensitive pairs of ionospheric negative (IN) combination:

$$
\left|\frac{1}{\gamma-1}\left(\lambda_{1} \cdot C S_{1}-\lambda_{2} \cdot C S_{2}\right)\right|<4.565 \cdot \sigma_{M V^{-}}=0.069 \mathrm{~m} / \mathrm{s}^{2}
$$

Insensitive pairs of ionospheric positive (IP) combination:

$$
\left|\frac{1}{2}\left(\lambda_{1} \cdot C S_{1}+\frac{1}{\gamma} \lambda_{2} \cdot C S_{2}\right)\right|<4.565 \cdot \sigma_{M V^{+}}=0.078 \mathrm{~m} / \mathrm{s}^{2}
$$

Insensitive pairs of ionospheric free (IF) combination:

$$
\left|\frac{1}{\gamma-1}\left(\gamma \lambda_{1} \cdot C S_{1}-\lambda_{2} \cdot C S_{2}\right)\right|<4.565 \cdot \sigma_{I F}=0.133 \mathrm{~m} / \mathrm{s}^{2}
$$

Insensitive pairs of wide-lane (WL) combination:

$$
\left|\frac{c}{f_{1}-f_{2}} \cdot \frac{f_{2}}{f_{1}}\left(C S_{1}-C S_{2}\right)\right|<4.565 \cdot \sigma_{W L}=0.159 \mathrm{~m} / \mathrm{s}^{2}
$$


Insensitive pairs of narrow-lane (NL) combination:

$$
\left|\frac{c}{f_{1}+f_{2}} \cdot \frac{f_{2}}{f_{1}}\left(C S_{1}+C S_{2}\right)\right|<4.565 \cdot \sigma_{N L}=0.076 \mathrm{~m} / \mathrm{s}^{2}
$$

In order to successfully detect all cycle slips, we must choose two independent combination for cycle-slip detection that their insensitive pairs do not overlap. For example, as shown in Figure 5, if we choose the IP and NL combination as MVs, the special cycle-slip pairs $(-1,1)$ cannot be detected. On the other hand, the IN and IP combination have an opposite signs of inclination and their inclination is completely orthogonal; that is, they do not share the insensitive pairs with each other.

In order to choose two optimal combination for cycle-slip detection, we evaluate the probability of MD for cycle-slip pairs of each linear combination. The evaluated probability of $\mathrm{MD}$ is shown in color map in Figure 6 . The cycle slips are easily detectable when the color area is blue, while it is difficult to detect when the color changes toward red. The insensitive cycleslip pairs for each individual combination have a large MD rate close to one. Therefore, we should employ at least two independent and complementary combinations to detect all cycle-slips. For example, the total probability of MD by the product of the MD rate of the IN and IP combination, as shown in Figure 7, is very small because each insensitive pair can surely be detected by the complementary and orthogonal MVs. Probability of MD for pair $(1,1)$ of $\mathrm{IN}$ combination is 0.174 . That is, the IN combination may fail to detect the pair $(1,1)$ with a probability of $17.4 \%$, whereas the pair $(1,1)$ has a MD probability of $4.3 \times 10^{-8}$ by the IP combination. Therefore, the total probability of MD is $7.5 \times 10^{-9}$ that is the product value of 0.174 and 4.3 $\times 10^{-8}$. In case of IN and IP combination, the maximum probability of MD is $7.5 \times 10^{-9}$ when the cycle-slip pair is $(1,1)$ or $(-1$, -1 ). The remaining pairs have a much smaller probability.

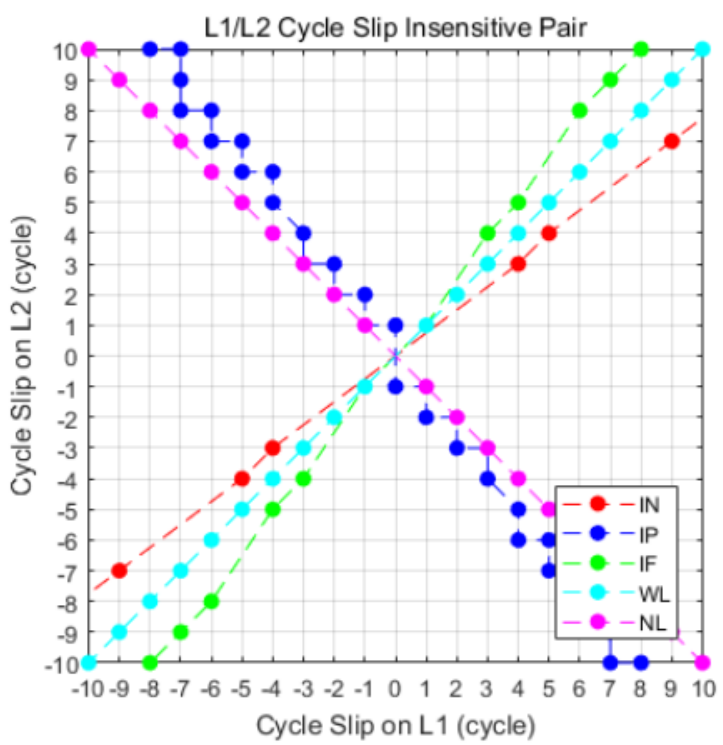

Figure 5. L1/L2 insensitive cycle-slip pairs of each combinations

Table 3. Maximum probability of missed detection by MV selection

\begin{tabular}{cccc}
\hline MV \#1 & MV \#2 & Total PMD & Cycle-Slip Pair \\
\hline IN & IP & $7.5 \times 10^{-9}$ & $(1,1)$ \\
IN & IF & 0.142 & $(1,1)$ \\
IN & WL & 0.174 & $(1,1)$ \\
IN & NL & $3.0 \times 10^{-8}$ & $(1,1)$ \\
IP & IF & $3.5 \times 10^{-8}$ & $(1,1)$ \\
IP & WL & $4.3 \times 10^{-8}$ & $(1,1)$ \\
IP & NL & 1.000 & $(-1,1)$ \\
IF & WL & 0.816 & $(1,1)$ \\
IF & NL & $1.4 \times 10^{-8}$ & $(1,1)$ \\
WL & NL & $1.7 \times 10^{-8}$ & $(1,1)$ \\
\hline
\end{tabular}


Table 3 summarizes the maximum probability of MD of other MV selections in a similar way. As can be seen from the results, the IN combination with IP combination has smaller MD probability at cycle-slip pair $(1,1)$ than other selections. The other various MV selections (IN + NL), (IP + IF), (IP + WL), (IF + NL), (WL + NL) also show sufficient performance to detect all cycle slips under MD probability $10^{-7}$; however, the cycle-slip detection using the IN and IP combination shows the best detection performance. Consequently, our detection method using the acceleration of the IN and IP combinations ensures highintegrity detection with a MD probability of $7.5 \times 10^{-9}$ under the desired total FA probability of $10^{-5}$. Though it can be applied only stationary observations, we verified that the IP combination can be optimally detect cycle-slip with the IN combination over the other combination including the MW pseudorange combination. Therefore, the proposed method with the optimal IN and IP combination is suitable for treating dual-frequency cycle slips on network-based RTK technique.

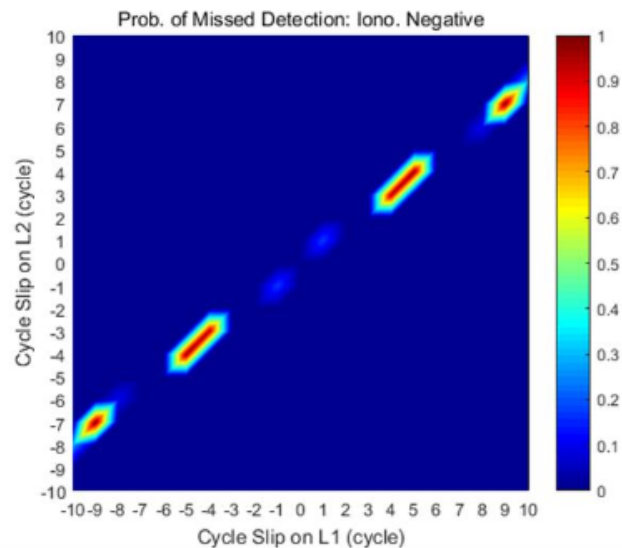

(a)

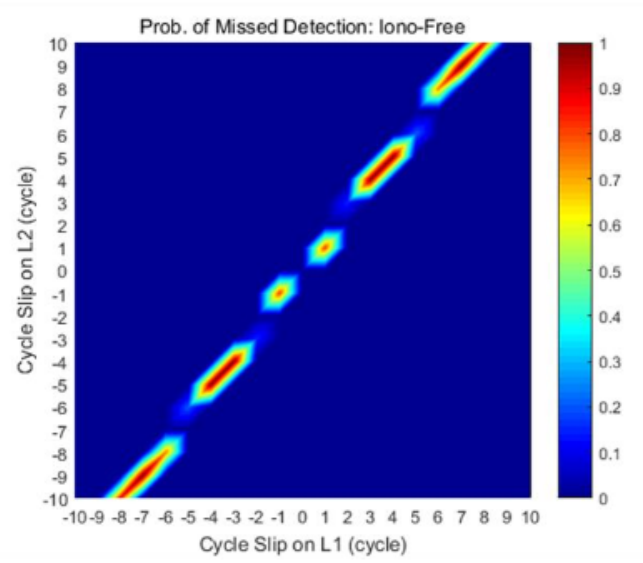

(c)

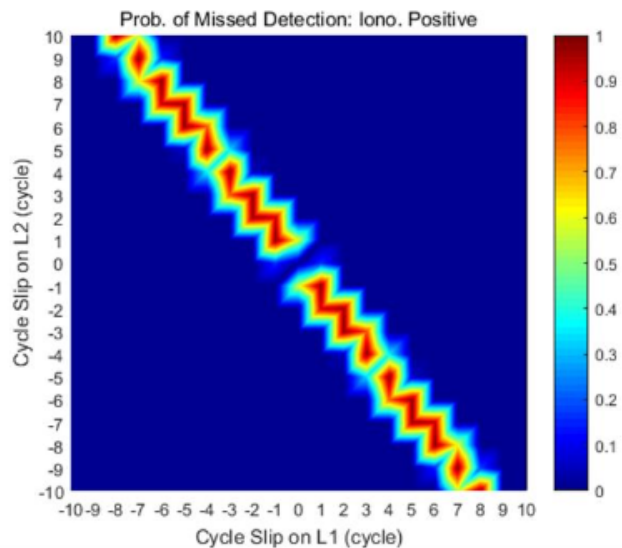

(b)

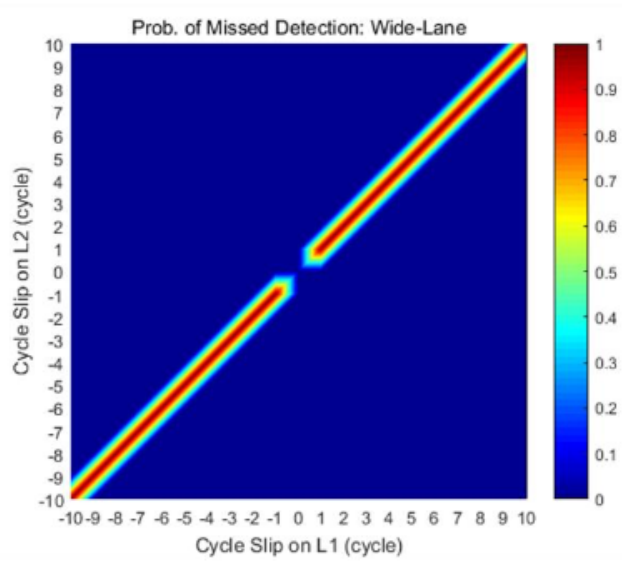

(d)

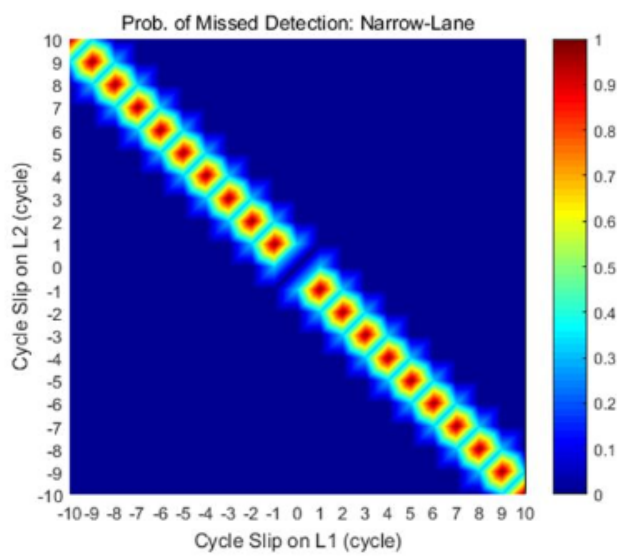

(e)

Figure 6. Probability of missed detection for cycle-slip pairs of each combination: (a) IN combination; (b) IP combination; (c) IF combination; (d) WL combination; (e) NL combination 


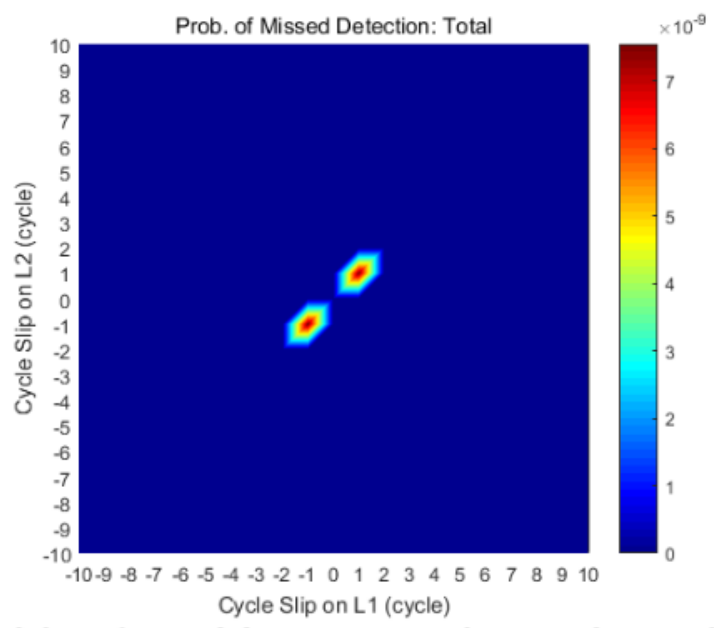

Figure 7. Total Probability of missed detection using the IN and IP combinations simultaneously

\section{ALGORITHM TEST RESULTS UNDER SEVERE IONOSPHERIC STORM CONDITIONS}

\section{Algorithm Test Environment}

The proposed algorithm using the IN and IP combination, which are optimal carrier-phase combinations, is verified using GPS carrier-phase measurements collected under a severe geomagnetic storm that occurred on 17 March 2015. The test environment is summarized in Table 4. We collected the GPS dual-frequency carrier phase with 1 -s intervals from the GANH $\left(37.72^{\circ} \mathrm{N}, 126.39^{\circ} \mathrm{E}\right)$ and $\mathrm{CHJU}\left(33.51^{\circ} \mathrm{N}, 126.53^{\circ} \mathrm{E}\right)$ stations of NGII in Korea. Their locations are shown in Figure 2 (b), and the baseline length between them is $467 \mathrm{~km}$. The maximum ionospheric disturbance was observed between 15:00:00 and 21:00:00 local time in the Korean region (UTC + 9). We conducted the algorithm validation test using the low-elevation data of GPS PRN 19, which are specifically affected by the ionospheric disturbance. Practically, cycle slips frequently occur in the low-elevation data owing to the low signal-to-noise ratio and the possibility of signal blockage. First, we confirmed that no real cycle slip occurred in these target data by pre-processing using Bernese GNSS software. Next, we forcibly inserted the simulated cycle slips, which are the typical insensitive pairs, every 100 epochs into the raw observations.

Table 4. Algorithm test environment

\begin{tabular}{|c|c|}
\hline Date & 17 March 2015 \\
\hline Time & UTC 06:00:00 11:59:59 (6 h) \\
\hline Interval & 1 second \\
\hline Baseline & GANH-CHJU (467 km) \\
\hline Receiver & Trimble NetR9 \\
\hline Antenna & Trimble Zephyr (TRM59800) \\
\hline Kp index & 8- (Daily maximum) \\
\hline
\end{tabular}

\section{Data Test Results}

The algorithm test results are summarized in Table 5, and the time series of MVs are illustrated in Figure 8. In this figure, the top subplot represents IN acceleration, and the bottom subplot represents IP acceleration. The blue line shows the MV and the dash-single-dotted magenta line indicates the threshold for cycle-slip detection. The red star points show the missed cycle slip, which correspond to the insensitive pairs for each combination.

As can be seen from the results, all of the cycle slips including the pair $(1,1)$ for which the probability of MD has the maximum value are correctly detected by the two independent and complementary MVs, IN and IP acceleration. For example, the cycle-slip pair $(5,4)$, one of the most challenging pairs to detect in many previous studies, is not detected by the IN combination but is successfully detected using the IP combination. In contrast, the insensitive pairs in the IP combination are apparently detected under the IN combination. 

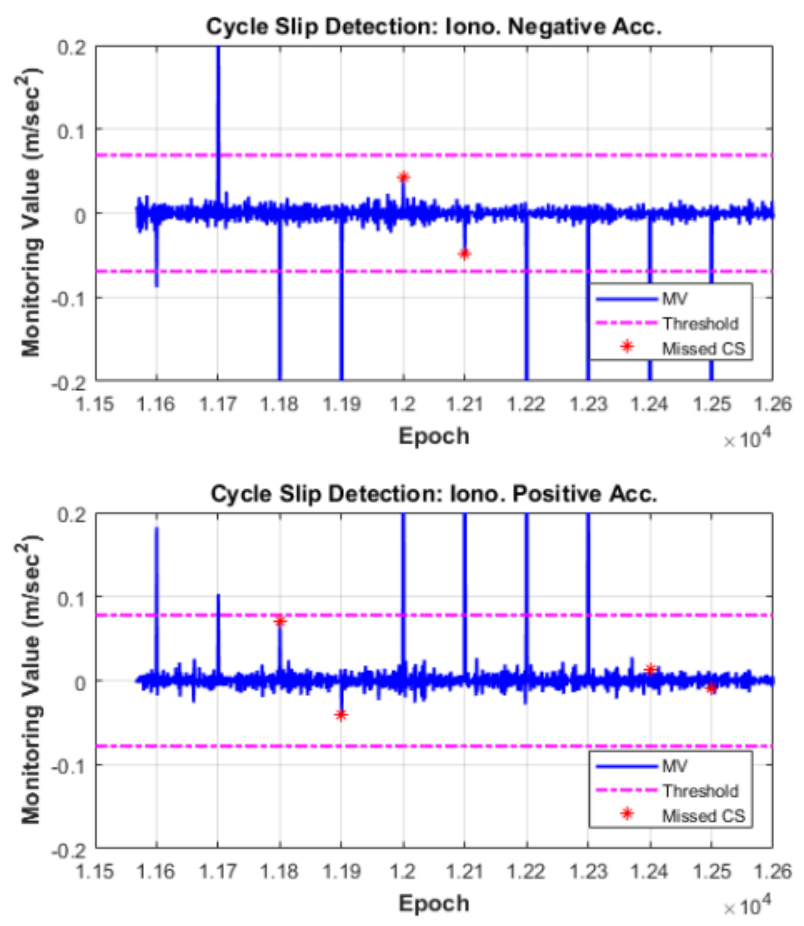

Figure 8. Cycle-slip detection results for PRN 19

Table 5. Summary of Cycle-slip detection results (any probability less than 10-100 is entered as zero)

\begin{tabular}{cccccccc}
\hline \multirow{2}{*}{ Epoch } & \multirow{2}{*}{ El ( ${ }^{\circ}$ ) } & $\begin{array}{c}\text { Inserted } \\
\text { Cycle Slip }\end{array}$ & $\begin{array}{c}\text { Total } \\
\text { PMD }\end{array}$ & \multicolumn{2}{c}{$\begin{array}{c}\text { Monitoring Value } \\
\text { (meter) }\end{array}$} & \multicolumn{2}{c}{ Detected? } \\
\cline { 5 - 8 } & & & & $\mathbf{M V}^{-}$ & $\mathbf{M V}^{+}$ & $\mathbf{M V}^{-}$ & $\mathbf{M V}^{+}$ \\
\hline 11600 & 5.99 & $(1,1)$ & $7.5 \times 10^{-9}$ & -0.088 & 0.183 & $\mathrm{O}$ & $\mathrm{O}$ \\
11700 & 6.67 & $(1,0)$ & $4.9 \times 10^{-51}$ & 0.291 & 0.103 & $\mathrm{O}$ & $\mathrm{O}$ \\
11800 & 7.36 & $(0,1)$ & $1.1 \times 10^{-92}$ & -0.385 & 0.070 & $\mathrm{O}$ & $\mathrm{X}$ \\
11900 & 8.05 & $(-1,1)$ & 0 & -0.669 & -0.041 & $\mathrm{O}$ & $\mathrm{X}$ \\
12000 & 8.74 & $(4,3)$ & 0 & 0.043 & 0.598 & $\mathrm{X}$ & $\mathrm{O}$ \\
12100 & 9.44 & $(5,4)$ & 0 & -0.049 & 0.771 & $\mathrm{X}$ & $\mathrm{O}$ \\
12200 & 10.14 & $(3,4)$ & 0 & -0.625 & 0.569 & $\mathrm{O}$ & $\mathrm{O}$ \\
12300 & 10.84 & $(4,5)$ & 0 & -0.708 & 0.732 & $\mathrm{O}$ & $\mathrm{O}$ \\
12400 & 11.55 & $(-3,4)$ & 0 & -2.389 & 0.013 & $\mathrm{O}$ & $\mathrm{X}$ \\
12500 & 12.26 & $(-4,5)$ & 0 & -3.075 & -0.010 & $\mathrm{O}$ & $\mathrm{X}$ \\
\hline
\end{tabular}

\section{CONCLUSIONS}

This paper proposed a cycle-slip detection algorithm using only dual-frequency carrier-phase stationary observations. In order to detect cycle slips with high-integrity performance, two complementary ionospheric combinations called the IN and IP combinations are employed. They can successfully detect all of the cycle slips since two L1/L2 combinations combine cycle slips with opposite signs for uniquely detecting insensitive pairs. We verified that the actual error distributions of the MVs under high ionospheric storm are sufficiently bounded and properly assumed at the $10^{-7}$-level probability of the normal Gaussian distribution from a theoretical analysis. The detection threshold is determined by the total desired probability of FA of $10^{-5}$. Consequently, our detection method ensures high-integrity performance with a probability of MD of $7.5 \times 10^{-9}$. The IN and IP combination shows the best detection performance than the other linear combinations. Finally, an algorithm verification test was conducted using actual data collected under a severe ionospheric storm. As a result, all artificially inserted cycle slips, including small cycle slips, were successfully detected. In summary, we demonstrated that the proposed method is suitable and optimal for monitoring dual-frequency cycle slips on network RTK systems, which should generate high-precision corrections with high-integrity information. 


\section{ACKNOWLEDGMENTS}

This research was supported by Basic Science Research Program through the National Research Foundation of Korea (NRF) funded by the Ministry of Science, ICT \& Future Planning (No. NRF-2017M1A3A3A02016230), contracted through by the Institute of Advanced Aerospace Technology at Seoul National University. The Institute of Engineering Research at Seoul National University provided research facilities for this work.

\section{REFERENCES}

[1] B. Park, "A Study on Reducing Temporal and Spatial Decorrelation Effect in GNSS Augmentation System: Consideration of the Correction Message Standardization," Ph.D. Thesis, Seoul National University, Seoul, Korea, 2008.

[2] B. Park and C. Kee, "The Compact Network RTK Method: An Effective Solution to Reduce GNSS Temporal and Spatial Decorrelation Error," J. Navig., vol. 63, no. 2, pp. 343-362, 2010.

[3] J. Song, "A Study on Improving Performance of Network RTK through Tropospheric Modeling for Land Vehicle Applications," Ph.D. Thesis, Seoul National University, Seoul, Korea, 2016.

[4] S. B. Bisnath, D. Kim, and R. B. Langley, "A New Approach to an Old Problem: Carrier-Phase Cycle Slips," GPS World, vol. 13, no. 4, pp. 42-49, 2001.

[5] J. Seo and T. Walter, "Future Dual-Frequency GPS Navigation System for Intelligent Air Transportation Under Strong Ionospheric Scintillation," IEEE Trans. Intell. Transp. Syst., vol. 15, no. 5, pp. 2224-2236, 2014.

[6] X. Luo, Z. Liu, Y. Lou, S. Gu, and B. Chen, "A Study of Multi-GNSS Ionospheric Scintillation and Cycle-Slip over Hong Kong Region for Moderate Solar Flux Conditions," Adv. Sp. Res., vol. 60, no. 5, pp. 1039-1053, 2017.

[7] G. Blewitt, "An Automatic Editing Algorithm for GPS data," Geophys. Res. Lett., vol. 17, no. 3, pp. 199-202, 1990.

[8] Y. Gao and Z. Li, "Cycle Slip Detection and Ambiguity Resolution Algorithms for Dual-Frequency GPS Data Processing," Mar. Geod., vol. 22, no. 3, pp. 169-181, 1999.

[9] Z. Liu, "A New Automated Cycle Slip Detection and Repair Method for a Single Dual-Frequency GPS Receiver," J. Geod., vol. 85, no. 3, pp. 171-183, 2011.

[10] C. Cai, Z. Liu, P. Xia, and W. Dai, "Cycle Slip Detection and Repair for Undifferenced GPS Observations Under High Ionospheric Activity," GPS Solut., vol. 17, no. 2, pp. 247-260, 2013.

[11] J. Song and C. Kee, "A New Approach for GNSS Frequency Selection Considering Detection of Cycle Slip Insensitive Pairs of Ionospheric Combination for Dual- Frequency Receivers," in Proceedings of the 28th International Technical Meeting of The Satellite Division of the Institute of Navigation (ION GNSS+ 2015), 2015, pp. 2681-2687.

[12] W. Liu, X. Jin, M. Wu, J. Hu, and Y. Wu, "A New Real-Time Cycle Slip Detection and Repair Method Under High Ionospheric Activity for a Triple-frequency GPS/BDS Receiver," Sensors, vol. 18, no. 2, p. 427, 2018.

[13] T. Zeng et al., "Real-Time Triple-Frequency Cycle Slip Detection and Repair Method Under Ionospheric Disturbance Validated with BDS Data," GPS Solut., vol. 22, no. 3, pp. 1-13, 2018.

[14] Z. Dai, S. Knedlik, and O. Loffeld, "Instantaneous Triple-Frequency GPS Cycle-Slip Detection and Repair," Int. J. Navig. Obs., vol. 2009, pp. 1-15, 2009.

[15] Y. Kim, J. Song, C. Kee, and B. Park, "GPS Cycle Slip Detection Considering Satellite Geometry based on TDCP/INS Integrated Navigation," Sensors, vol. 15, no. 10, pp. 25336-25365, 2015.

[16] D. Kim, J. Song, S. Yu, C. Kee, and M. Heo, "A New Algorithm for High-Integrity Detection and Compensation of Dual-Frequency Cycle Slip under Severe Ionospheric Storm Conditions," Sensors, vol. 18, no. 11, p. 3654, 2018.

[17] S. Pullen, "Augmented GNSS: Fundamentals and Keys to Integrity and Continuity," in ION GNSS 2011 Tutorial, 2011.

[18] B. DeCleene, "Defining Pseudorange Integrity - Overbounding," in Proceedings of the 13th International Technical Meeting of the Satellite Division of The Institute of Navigation (ION GPS 2000), 2000, no. September, pp. 1916-1924.

[19] T. R. Schempp and A. L. Rubin, "An Application of Gaussian Overbounding for the WAAS Fault Free Error Analysis," in Proceedings of the 15th International Technical Meeting of the Satellite Division of The Institute of Navigation (ION GPS 2002), 2002, no. September, pp. 766-772.

[20] R. Dach, S. Lutz, P. Walser, and P. Fridez, Bernese GNSS Software Version 5.2. Astronomical Institute, University of Bern, 2015.

[21] M. Olynik, M. Petovello, M. Cannon, and G. Lachapelle, "Temporal Impact of Selected GPS Errors on Point Positioning," GPS Solut., vol. 6, no. 1-2, pp. 47-57, 2002.

[22] C. Altmayer, "Enhancing the integrity of integrated GPS/INS systems by cycle slip detection and correction," in Proceedings of the IEEE Intelligent Vehicles Symposium 2000, 2000, pp. 174-179. 